\title{
A fracture locus for a 10 volume-percent V95/SiC metal matrix composite at the near-solidus temperature
}

\author{
D. I. Vichuzhanin ${ }^{1, \dagger}$, S. V. Smirnov ${ }^{1}$, A. V. Nesterenko', A. S. Igumnov ${ }^{2}$ \\ ${ }^{\dagger}$ mmm@imach.uran.ru
}

\author{
${ }^{1}$ Institute of Engineering Science, Ural Branch of RAS, 34 Komsomolskaya str., Yekaterinburg, 620219, Russia \\ ${ }^{2}$ Institute of Mathematics and Mechanics, Ural Branch of RAS, 16 S. Kovalevskaya str., Yekaterinburg, 620990, Russia
}

\begin{abstract}
A fracture locus for a $10 \mathrm{vol} \% \mathrm{~V} 95 / \mathrm{SiC}$ metal matrix composite at near-solidus temperature is obtained from experiments. The fracture locus determines a functional dependence of the value of ultimate metal ductility on the stress state characteristics. To study ultimate metal ductility, test specimens are deformed under prevailing compressive stresses. Tests for cylindrical specimen compression, bell-shaped specimen compression, cylindrical specimen torsion and for supported extrusion of the bottoms of thick-walled thin-bottomed cups are used. The tests are simulated by the finite element method to evaluate the stress-strain state in the fracture region. An isotropic elastic-plastic strain-hardening model is used as the material to be deformed. The forming tools are considered rigid. The strain state is viewed as axisymmetric; therefore, only a half of the longitudinal section of the specimen is simulated. The fracture locus makes it possible to evaluate the ultimate ductility of the composite as a function of the stress triaxiality factor $k$ and the Lode-Nadai coefficient $\mu_{\text {. }}$. The stress triaxiality factor characterizes the relative level of normal stresses. The Lode-Nadai coefficient characterizes the form of the stress state. The combination of these parameters uniquely characterizes the stress state under plastic deformation. The fracture locus can be used in the ranges $-1.2<k<0.4$ and $0<\mu_{\sigma}<+1$. The obtained fracture locus is applicable to the evaluation of the damage accumulated in workpieces for machine parts and structural components made of this composite. Thus a workpiece production process can be optimized.
\end{abstract}

Keywords: metal matrix composite, fracture locus.

УДК 621.7.011+569.442.23+620.163.2

\section{Диаграмма предельной пластичности металломатричного композита B95/SiC с содержанием частиц SiC 10 об.\% при околосолидусной температуре}

\author{
Вичужанин Д. И. ${ }^{1, \dagger}$, Смирнов С. В. ${ }^{1}$, Нестеренко А. В. ${ }^{1}$, Игумнов А.С. ${ }^{2}$ \\ ${ }^{1}$ Институт машиноведения УрО РАН, ул. Комсомольская 34, Екатеринбург, 620049, Россия \\ ${ }^{2}$ Институт математики и механики УрО РАН, ул. Софьи Ковалевской 16, Екатеринбург, 620990, Россия
}

На основании результатов экспериментов получена диаграмма предельной пластичности металломатричного композита B95/SiC с содержанием частиц $\mathrm{SiC} 10$ об.\% при околосолидусной температуре, которая устанавливает функциональную связь между величиной предельной пластичности и характеристиками напряженного состояния. Для исследований предельной пластичности композита использовали такие виды испытаний, при которых образец деформируется в условиях преобладающих сжимающих напряжений: сжатие цилиндрических образцов, сжатие образцов типа «колокольчик», выдавливание с подпором донышка толстостенного стаканчика с утонением на донышке, кручение цилиндрических образцов. Для оценки напряженно - деформированного состояния образцов в месте разрушения выполнено математическое моделирование испытаний методом конечных элементов. В качестве материала деформируемых образцов принималась изотропная упруго - пластическая модель деформационного упрочнения. Материал деформирующего инструмента рассматривался как абсолютно жесткое тело. Так как расчеты выполнены в предположении осесимметричного деформированного состояния в очаге деформации, то моделировалась только половина сечения образца. Диаграмма позволяет оценивать предельную пластичность композита в зависимости от коэффициента напряженного состояния $k$ и коэффициента Лоде-Надаи $\mu_{\sigma}$. Коэффициент $k$ характеризует относительный уровень нормальных напряжений, а коэффициент $\mu_{\sigma}-$ вид напряженного состояния. Совокупность коэффициентов $k$ и $\mu_{\sigma}$ однозначно характеризует напряженное состояние при пластической деформации. Область использования диаграммы ограничена интервалами изменений $-1,2<k<0,4$ и $0<\mu_{\sigma}<+1$. Полученная диаграмма предельной пластичности в дальнейшем может быть использована для оценки накопленной в процессе пластического формоизменения поврежденности композита, которая в свою очередь может быть использована для оптимизации технологического процесса получения заготовок деталей конструкционного назначения.

Ключевые слова: металломатричный композит, диаграмма предельной пластичности. 


\section{1. Введение}

Металломатричный композит (ММК) представляет собой конструкционный материал, состоящий из пластичной металлической матрицы и высокопрочных частиц наполнителя. В качестве материала матрицы могут выступать различные металлы, например: магний, титан, алюминий и их сплавы. В качестве частиц наполнителя часто используют карбид кремния [1], карбид бора [2], карбид титана [3], графит [4], углеродные нанотрубки [5], графен [6]. Добавление частиц наполнителя в ММК позволяет улучшить физико-механические свойства композита [7] по сравнению со свойствами материала матрицы при достаточно низкой стоимости композита. Таким образом, ММК является перспективным конструкционным материалом, использующимся в аэрокосмической, автомобильной, электронной и многих других отраслях промышленности.

Проанализируем, какие проблемы ММК наиболее популярны у исследователей. Большинство научных статей посвящено исследованию структуры ММК (состав матрицы и упрочняющих частиц, содержание и размер упрочняющих частиц) после изготовления ММК, различным аспектам изготовления по жидкофазным и порошковым технологиям и их влиянию на физико-механические свойства [8-10]. Значительное число публикации относится к исследованию процессов деформирования ММК и формированию комплекса физико-механических свойств в результате деформационных воздействий [11-13].

Известно, что при пластической деформации протекают процессы возникновения и развития дефектов сплошности в виде микропор и микротрещин, а также процессы залечивания микроповрежденности. Прогнозировать развитие поврежденности, а также залечивания и ускорить процесс выбора путей оптимизации способов и режимов деформационной обработки можно с помощью моделей механики поврежденности [14-15]. Одним из основных методологических выводов, следующих из положений механики поврежденности, является то, что по уровню стандартных механических характеристик (сужение, удлинение, временное сопротивление разрыву и др.) нельзя корректно судить о деформируемости металла при обработке давлением. Это связано с тем, что пластичность металла зависит от напряженного состояния, и эта связь, зачастую, является неоднозначной. Кроме того, напряженное состояние при испытаниях механических свойств и в технологическом процессе, как правило, существенно различается. Таким образом, определение предельных степеней деформации ММК до разрушения, а также уровня накопленной поврежденности в результате деформационных воздействий является чрезвычайно актуальной задачей.

Существует большое количество моделей накопления деформационной поврежденности металлических материалов, критериев ее оценки и экспериментальных методик оценки пластических свойств. Достаточно подробный обзор этих исследований приведен в работах [16-17]. В рамках данной работы использовали линейную феноменологическую модель разрушения
В. Л. Колмогорова [14-15], в которой под поврежденностью понимается безразмерный скалярный параметр $\omega$. В начальный момент деформации $\omega=0$, в процессе деформации поврежденность накапливается и в момент разрушения $\omega=1$. Величина поврежденности прямо пропорциональна накопленной степени деформации сдвига и обратно пропорциональна предельной пластичности материала:

$$
\omega=\int_{0}^{\Lambda} \frac{d \Lambda}{\Lambda_{f}\left(k, \mu_{\sigma}\right)},
$$

где $\Lambda$ - степень деформации сдвига; $\Lambda_{f}\left(k, \mu_{\sigma}\right)-$ степень деформации сдвига, накопленная к моменту разрушения (характеристика предельной пластичности материала) в условиях монотонного деформирования при постоянных на всем процессе деформирования величинах $k$ и $\mu_{\sigma}$. Величина $\Lambda_{f}\left(k, \mu_{\sigma}\right)$ является функцией коэффициента напряженного состояния $k$ и коэффициента Лоде-Надаи $\mu_{\sigma}$. Совокупность коэффициентов $\mathrm{k}$ и $\mu \sigma$ однозначно характеризует напряженное состояние при пластической деформации. Графическая интерпретация функции $\Lambda_{f}\left(k, \mu_{\sigma}\right)$ называется диаграммой предельной пластичности материала.

Одним из важнейших технологических параметров пластического деформирования металлов является температура деформации, от которой во многом зависит возможность осуществления формоизменения без разрушения. Для ММК на основе алюминиевых сплавов перспективным представляется увеличение температур технологической деформации вплоть до околосолидусных, что в соответствии с результатами исследований [18] приводит к снижению интенсивности образования микропористости на частицах $\mathrm{SiC}$, повышению равномерности их распределения в матрице и, как следствие, улучшению их деформационных свойств [19-20].

Цель исследования - построение диаграммы предельной пластичности металломатричного композита на основе сплава В95 с содержанием наполнителя в виде частиц $\mathrm{SiC} 10$ об. \% при температуре $570^{\circ} \mathrm{C}$ Диаграмма предельной пластичности в дальнейшем может быть использована для оценки накопленной в процессе пластического формоизменения поврежденности композита, которая в свою очередь может быть использована для оптимизации технологического процесса получения заготовок деталей конструкционного назначения.

\section{2. Материал и методика исследования}

Исследованный ММК изготовлен во Всероссийском научно-исследовательском институте авиационных материалов (ВИАМ), имеет матрицу из высокопрочного сплава В95 системы $\mathrm{Al}-\mathrm{Zn}-\mathrm{Mg}-\mathrm{Cu}$. ММК содержит 10 об. \% наполнителя в виде частиц $\mathrm{SiC}$. Композит получен по технологии порошковой металлургии путем спекания смеси порошков алюминиевого сплава и карбида кремния.

Для исследований предельной пластичности композита использовали комплекс испытаний, предложенный в [16]: растяжение гладких (без выточки) цилиндрических образцов, растяжение цилиндрических образцов 
с концентраторами напряжений (выточкой на боковой поверхности), сжатие цилиндрических образцов, сжатие и растяжение образцов типа «колокольчик», выдавливание с подпором и без подпора донышка толстостенного стаканчика с утонением на донышке, а также кручение цилиндрических образцов.

По результатам испытаний на растяжение различных видов образцов было установлено, что материал в этих условиях разрушается хрупко. Разрушенные образцы практически не имеют остаточной деформации. Поэтому было принято решение исследовать пластичность композита только в области сжимающих напряжений. При этом воспользовались результатами следующих видов испытаний: сжатие цилиндрических образцов, кручение цилиндрических образцов, сжатие образцов типа «колокольчик», выдавливание с подпором донышка толстостенного стаканчика с утонением на донышке. Данные виды испытаний позволяют в широком диапазоне варьировать в процессе испытаний значения коэффициентов $k$ и $\mu_{\sigma}$.

Испытания проведены на сервогидравлической испытательной машине INSTRON 8801, снабженной нагревательной печью при температуре $570^{\circ} \mathrm{C}$. Температура солидуса композита равна $593,6^{\circ} \mathrm{C}$ [21]. Температура образца контролировалась хромель-копелевой термопарой.

Цилиндрические образцы с гладкой боковой поверхностью имели начальные диаметр $D_{0}=6 \pm 0.05$ мм и высоту $H_{0}=9+0.05$ мм. Для уменьшения трения на контакте деформируемого образца с инструментом использовали графитовую смазку. Испытания проводили вплоть до момента появления трещины на боковой поверхности образца.

Цилиндрические образцы для испытаний на кручение имели начальный диаметр $D_{0}=5 \pm 0.05$ мм и длину $L_{0}=15 \pm 0.05$ мм. Степень деформации по сечению образца распределена не равномерно. На оси симметрии она равна нулю и является максимальной на поверхности образца. При этом разрушение образца, как правило, начинается на его поверхности. Степень деформации сдвига на поверхности образца определяли по формуле [15]:

$$
\Lambda=\operatorname{tg} \varphi_{1}-\operatorname{tg} \varphi_{0}
$$

где $\varphi_{0}$ и $\varphi_{1}-$ углы наклона риски, напечатанной типографским способом на поверхности образца, к образующей до испытания и после него.

Образцы типа «колокольчик» позволяют исследовать пластичность материала в условиях близких к схеме плоского напряженного состояния $\mu_{\sigma} \approx 0$. При этом, в испытаниях на сжатие в месте разрушения преобладают нормальные сжимающие напряжения, в испытаниях на растяжение - нормальные растягивающие напряжения. Подробное описание образца и методика его испытания на сжатие приведены в [17].

Испытания на выдавливание донышка толстостенного стаканчика позволяют исследовать пластичность материала в условиях, когда коэффициент Лоде-Надаи $\mu_{\sigma} \approx+1$. Для создания сжимающих напряжений в очаге деформации использовали подпор. Подробное описание образца, оснастка для испытаний и методика испытаний приведены в [16].
Так как в процессе испытания трудно определить момент разрушения образца, то было выполнено некоторое количество испытаний каждого вида образцов, при этом образцы испытывались на разные степени деформации. После каждого испытания образец осматривался на предмет появления трещины. Всего было проведено по три серии испытаний каждого вида образцов. За степень деформации в момент разрушения приняли ее среднее арифметическое значение по результатам трех испытаний, в которых было достигнуто разрушение образца.

При испытаниях цилиндрических образцов на сжатие, сжатие образцов типа «колокольчик», выдавливание с подпором донышка толстостенного стаканчика напряженно-деформированное состояние образцов в месте разрушения определяли с помощью математического моделирования методом конечных элементов в пакете ANSYS 15.0. Для материала деформируемых образцов принималась изотропная упруго-пластическая модель деформационного упрочнения (MISO). Материал деформирующего инструмента рассматривался как жесткое тело. Так как расчеты выполнены в предположении осесимметричного деформированного состояния в очаге деформации, то моделировалась только половина сечения образца. Для моделирования использовали конечные элементы PLANE 183. Величину трения между деформирующим инструментом и деформируемым материалом рассчитывали с использованием закона трения по Зибелю. Величину коэффициента трения определяли методом осадки двух образцов, который приведен в [22]. По результатам исследований трения коэффициент трения установлен равным 0,29. Для оценки адекватности результатов моделирования выполнено сравнение усилия деформирования в процессе испытания с усилием, полученным в результате моделирования. Установлено, что отличия данных величин не превышают 6,3\%.

По результатам моделирования в месте разрушения образца получены зависимости коэффициента напряженного состояния $k$ и коэффициента Лоде-Надаи $\mu_{\sigma}$ от степени деформации сдвига $\Lambda$. Зависимости построены от начала процесса деформации вплоть до момента разрушения.

\section{3. Результаты исследования}

По результатам испытаний на сжатие цилиндрических образцов определили зависимость сопротивления деформации $\sigma$ от степени деформации сдвига $\Lambda$ (Рис. 1 ). В дальнейших исследованиях данную зависимость использовали для моделирования процессов испытаний методом конечных элементов.

По результатам испытаний на кручение определили среднюю арифметическую степень деформации сдвига в момент разрушения, которая равна $\Lambda=0,038$.

На Рис. 2 приведены фотографии образца типа колокольчик и образца в виде толстостенного стаканчика с утонением на донышке до и после испытания. Для наглядности образцы разрезаны по оси симметрии. В процессе испытания образцов типа колокольчик деформация локализуется в области наименьшего сечения, 
в этой же области происходит и разрушение (правая часть Рис. 2a). При испытании образцов в виде толстостенного стаканчика разрушение происходит на контакте образца с подпором на некотором расстоянии от оси симметрии.

В качестве примера на Рис. 3 приведена конечно-элементная модель и результаты моделирования испытания на сжатие образца типа колокольчик.

\section{4. Идентификация диаграммы пластичности}

Под идентификацией диаграммы предельной пластичности подразумевается определение неизвестных эмпирических коэффициентов, входящих в функцию предельной пластичности $\Lambda_{f}\left(k, \mu_{\sigma}\right)$. Для идентификации диаграммы пластичности исследованного композита воспользовались формулой, предложенной в [17].

Неизвестные коэффициенты функции $\Lambda_{f}\left(k, \mu_{\sigma}\right)$ определяли, воспользовавшись результатами моделирования испытаний. При этом, для каждого из испытаний выполняли расчет поврежденности по формуле (1). Так как функция $\Lambda_{f}\left(k, \mu_{\sigma}\right)$ определяется в условиях монотонного деформирования, то для соблюдения этого условия воспользуемся следующим приемом. Условно разделим истории деформирования на 100 равных участков. В пределах одного участка деформацию будем считать монотонной. Тогда в соответствие с принципом линейного суммирования поврежденности формулу (1) можно записать в виде:

$$
\omega=\sum_{i=1}^{100} \Delta \omega_{i}=\sum_{i=1}^{100} \Delta \Lambda_{i} / \Lambda_{f_{i}}=1
$$

где $i$ - номер монотонного участка деформирования.

Рассчитав для каждого из испытаний величину поврежденности по формуле (3) можно выполнить минимизацию функционала квадратичных отклонений поврежденности от 1:

$$
\sum_{j=1}^{4}\left(1-\omega_{j}\right)^{2} \rightarrow \min
$$

где $j$ - номер вида испытания.

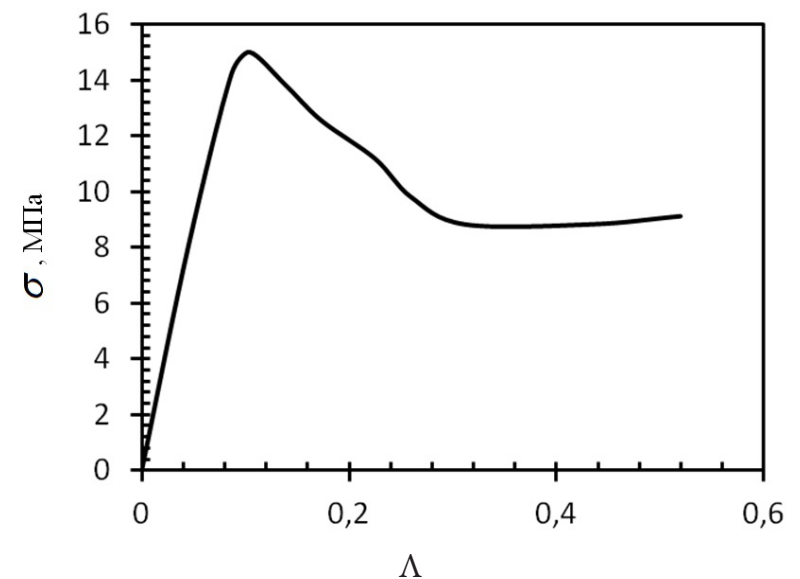

Рис. 1. Зависимость сопротивления деформации $\sigma$ от степени деформации сдвига $\Lambda$.

Fig. 1. True stress $\sigma$ as dependent on shear strain $\Lambda$.
Минимизацию функционала (4) выполнили путем варьирования неизвестных коэффициентов, входящих функцию $\Lambda_{f}\left(k, \mu_{\sigma}\right)$ и соответственно в формулу (3), методом Хука-Дживса [23]. Для того, чтобы не попасть в локальный минимум функционала (4), процедура ХукаДживса была выполнена не для одной начальной точки поиска, а для некоторого их множества.

На Рис. 4 приведена диаграмма предельной пластичности исследованного металломатричного композита B95/SiC с содержанием частиц $\mathrm{SiC} 10$ об.\%. Так как в условиях растягивающих напряжений исследованный композит разрушается хрупко, то диаграмма получена в интервалах изменений значений $-1,2<k<0,4$ и $0<\mu_{\sigma}<+1$.

\section{5. Заключение}

На основании результатов экспериментов получена диаграмма предельной пластичности металломатричного композита B95/SiC с содержанием частиц $\mathrm{SiC} 10$ об.\% при околосолидусной температуре, которая устанавливает функциональную связь между величиной предельной пластичности и характеристиками напряженного состояния. Область использования диаграммы ограничена интервалами изменений $-1,2<k<0,4$ и $0<\mu_{\sigma}<+1$.

Полученная диаграмма предельной пластичности может быть использована для оценки накопленной в процессе пластического формоизменения поврежденности композита, которая в свою очередь может быть использована для оптимизации технологического процесса получения заготовок деталей конструкционного назначения.

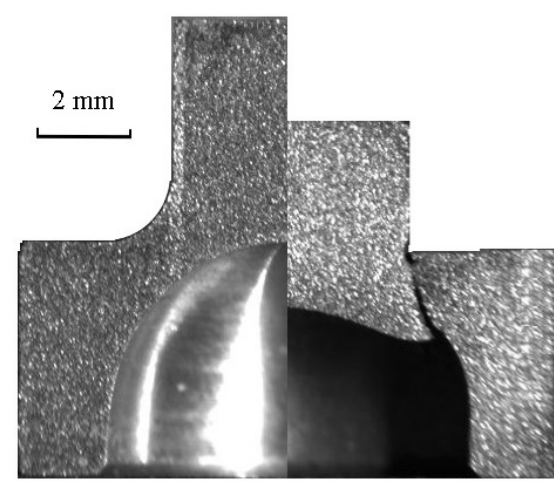

a

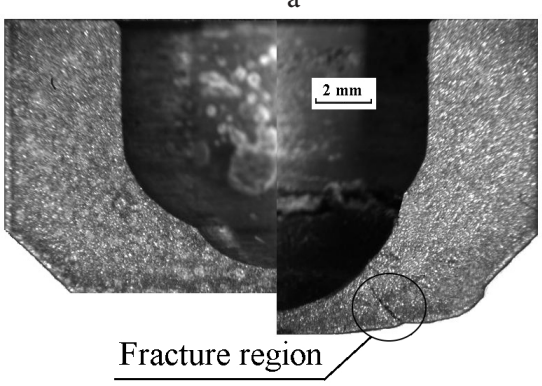

b

Рис. 2. Фотографии образца типа колокольчик (а) и образца толстостенного стаканчика с утонением на донышке (b) до и после испытания.

Fig. 2. Photographs of a bell-shaped specimen (a) and thick-walled cup with a thin bottom (b) before and after testing. 


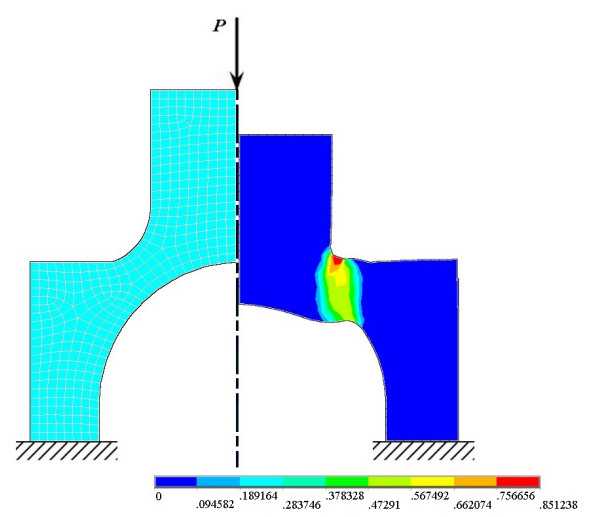

a

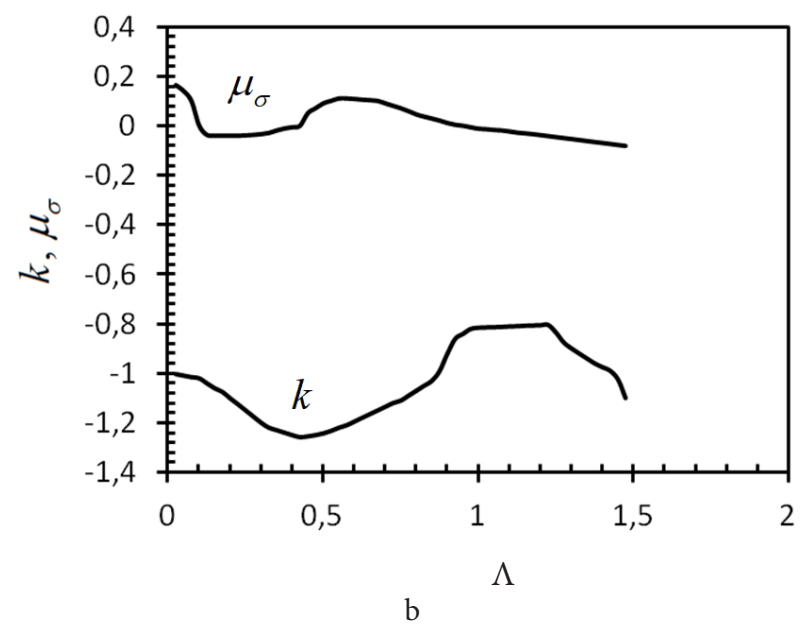

Рис. 3. Конечно-элементная модель испытания на сжатие образца типа колокольчик и распределение эквивалентных пластических деформаций $\varepsilon$ в момент разрушения (а), зависимости $k$ и $\mu_{\sigma}$ от $\Lambda$ в месте разрушения при испытании на сжатие образца типа колокольчик (b).

Fig. 3. A finite element model of the compression test of a bell-shaped specimen and equivalent plastic strains $\varepsilon$ at the fracture onset (a), the $k-\Lambda$ and $\mu_{\sigma}-\Lambda$ curves at the point of fracture for the compression test of a bell-shaped specimen (b).

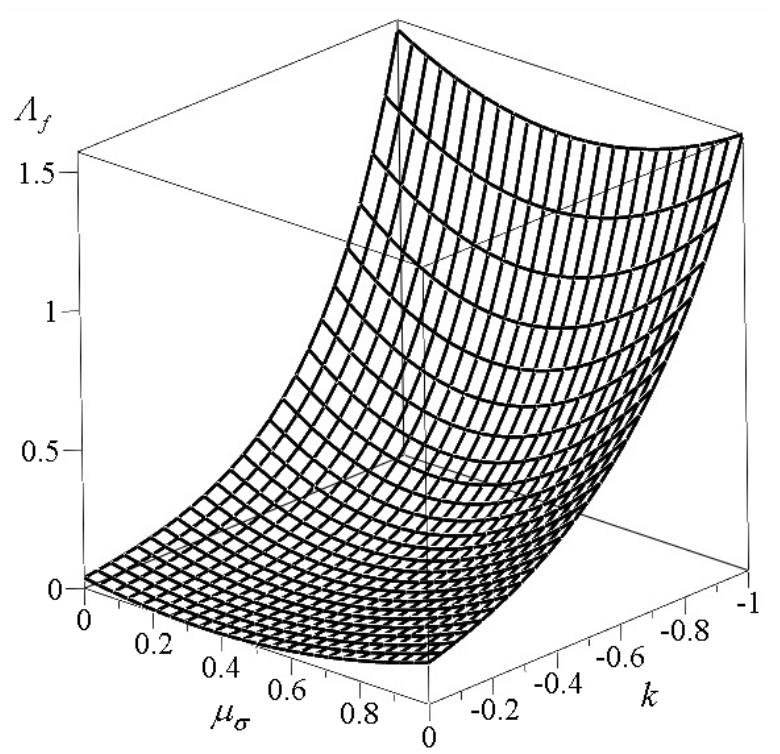

Рис. 4. Диаграмма пластичности металломатричного композита $\mathrm{B} 95 / \mathrm{SiC}$ с содержанием частиц $\mathrm{SiC} 10$ об.\%.

Fig. 4. A fracture locus for the 10 vol.\% V95/SiC metal matrix composite.
Благодарность/Acknowledgements. В части проведения экспериментов работа выполнена при поддержке гранта РНФ № 14-19-01358. Методика исследования пластичности была разработана в рамках темы государственного задания ИМАШ УрО РАН (№ 0391-2016-0004). Испьтания проведень с использованием оборудования ЦКП «Птастометрия» Института машиноведения УрО РАН. При проведении моделирования был использован суперкомпьютер «Уран» ИММ УрО РАН./This work was supported by the Russian Science Foundation (grant No. 14-19-01358) in the part of testing. The method for studying ductility was developed within the context of the theme of the state assignment for the Institute of Engineering Science, UB RAS (No. 0391-2016-0004). The tests were performed on the equipment installed at the Plastometriya collective use center affiliated to the Institute of Engineering Science, UB RAS. The finite element calculations were performed using the Uran supercomputer of IMM UB RAS.

\section{Литература/References}

1. A.A. El-Daly, M. Abdelhameed, M. Hashish, W. M. Daoush. Mat. Sci. Eng, A. 559, 384-393 (2013), DOI: $10.1016 /$ j.msea.2012.08.114

2. H. Yang, T. D. Topping, K. Wehage, L. Jiang, E. J. Lavemia, J.M. Schoenung. Mat.Sci. Eng, A. 616, 35-43 (2014), DOI: 10.1016/j.msea.2014.07.079

3. A. Lekatou, A.E. Karantzalis, A. Evangelou, V. Gousia, G. Kaptay, Z. Gacsi, P. Baumli, A. Simon. Mat. Des. 65, 1121 - 1135 (2015), DOI: 10.1016/j.matdes.2014.08.040

4. H. Kurita, T. Miyazaki, A. Kawasaki, Y. Lu, J-F. Silvain. Composites Part A. 73, 125 - 131 (2015), DOI: 10.1016/j. compositesa.2015.03.013

5. I. Alfonso, O. Navarro, O. Navarro, j. Vargas, A. Beltran, C. Aguilar, G. Gonzalez, I. A. Figueroa. Compos. Struct. 127, 420-425 (2015), DOI: 10.1016/j. compstruct.2015.03.032

6. R. Perez-Bustamante, D. Bolanos-Morales, J. BonillaMartinez, I. Estrada-Guel, R. Martinez-Sanchez. J. Alloys Compd. 615, 578-582 (2014), DOI: 10.1016/j. jallcom.2014.01.225

7. M. Rosso. J. Mater. Process. Technol. 175 (1), 364-365 (2006), DOI: 10.1016/j.jmatprotec.2005.04.038

8. J. Wozniak, B. Adamczyk-Cieslak, M. Kostecki, K. Broniszewski, W. Bochniak, A. Olszyna. Composites Part B. 77, $100-104$ (2015), DOI: 10.1016/j. compositesb.2015.03.018

9. S.N. Alhajeri, K. J. Al-Fadhalah, A.I. Almazrouee, T.G. Langdon. Mater. Charact. 118, 270-278 (2016), DOI: $10.1016 /$ j.matchar.2016.06.003

10. M.R. P. Fernandes, A.E. Martinelli, A.N. Klein, G. Hammes, C. Binder, R.M. Nascimento. Powder Technol. 305, 673-678 (2017), DOI: 10.1016/j. powtec.2016.10.053

11. Q. Hu, H. Zhao, F. Li. Mater. Sci. Eng., A. 680, 270-277 (2017), DOI: 10.1016/j.msea.2016.10.090

12. J. Jiang, Y. Wang. Mater. Des. 79, $32-41$ (2015), DOI: 10.1016/j.matdes.2015.04.040

13. M.S. Arab, N.E. Mahallawy, F. Shahata, M.A. Agwa. 
Mater. Des. 64, 280-286 (2014), DOI: 10.1016/j. matdes.2014.07.045

14. V.L. Kolmogorov. Stresses, strains, destruction. Moscow, Metallurgiia. (1970) 229 p. (in Russian) [В. Л. Колмогоров. Напряжения, деформации, разрушение. Москва, Металлургия. 1970. 229 с]

15. A. A. Bogatov, O.I. Mizhiritskii, S. V. Smirnov. Resource of metal plasticity under metal forming. Moscow, Metallurgiia. (1984) 144 p. (in Russian) [А. А. Богатов, О.И. Мижирицкий, С.В.Смирнов. Ресурс пластичности металлов при обработке давлением. Москва, Металлургия. 1984. 144 c]

16. S. V. Smirnov, D.I. Vichuzhanin A.V. Nesterenko . PNRPU Mechanics Bulletin. 3 (2015). (in Russian) [C. В. Смирнов, Д.И. Вичужанин, А.В. Нестеренко. Вестник ПНИПУ. Механика. 3 (2015).]

17. S. Smirnov, D. Vichuzhanin, A. Nesterenko, A. Smirnov, N. Pugacheva, A. Konovalov. Int. J. Mater. Form. (2016) (in press), DOI: 10.1007/s12289-016-1323-6
18. R. Rahmani Fard,.F. Akhlaghi. J. Mater. Process. Technol. 187-188, $433-436$ (2007) DOI: 10.1016/j. jmatprotec.2006.11.077

19. H. Iwasaki,M. Takeuchi, T. Mori, M. Mabuchi, K. Higashi. Scripta Metall. Mater. 31 (3), 255-260 (1994) DOI: 10.1016/0956-716X(94)90279-8

20. M. Mabuchi, K. Higashi, T.G. Langdon. Acta Metall. Mater. 42 (5), $1739-1745$ (1994). DOI: 10.1016/09567151(94)90384-0

21. N.B. Pugacheva, N.S. Michurov, E.I. Senaeva, T.M. Bykova. Phys Met Metallography. 117 (11), 1188 - 1195 (2016). DOI: 10.1134/S0031918X16110119

22. A.P. Grudev, Yu.V. Zilberg, V.T. Tilik. Friction and lubricants in metal forming. Moscow, Metallurgiia. (1982) 312 p. (in Russian) [А.П. Грудев, Ю.В. Зильберг, B.Т. Тилик. Трение и смазки при обработке металлов давлением. Москва, Металлургия. 1982. 312 с]

23. R. Hooke, T.A. Jeeves. JACM. 8, 212-229 (1961), DOI: $10.1145 / 321062.321069$ 